\title{
Avaliação Léxico-Sintática de Atividades Escritas em Algoritmo Genético e Processamento de Linguagem Natural: Um Experimento no ENEM
}

Title: Lexical-Syntactic Evaluation of written activities based on Genetic Algorithm and Natural Language Processing: An experiment on ENEM

Jário Santos

Instituto de Computação (IC) Universidade Federal de Alagoas (UFAL) jjsj@ic.ufal.br

\section{Ranilson Paiva}

Instituto de Computação (IC)

- Universidade Federal de

Alagoas (UFAL)

roap@ic.ufal.br
Ig Ibert Bittencourt

Instituto de Computação (IC) -

Universidade Federal de Alagoas

(UFAL)

ig.ibert@ic.ufal.br

\begin{abstract}
Resumo A Educação Online vem crescendo bastante nos últimos anos e diversos fatores propiciam esse crescimento. Atualmente, existem diversas tecnologias que dão apoio à Educação Online. Nesse contexto, temos milhões de usuários, produzindo uma imensa quantidade de dados educacionais, que podem, de certa forma, sobrecarregar professores e tutores, deixando o aluno na espera por feedback. Com a utilização de técnicas computacionais de correção automática, têm-se uma fluidez maior de atividades e diminuição relevante na sobrecarga de trabalho do professor, deixando-o livre para o desenvolvimento de outras atividades. Este trabalho apresenta um Analisador Ortográfico-Gramatical para avaliação automática de Atividades escritas em Língua Portuguesa com uso de Algoritmos Genéticos e Processamento de Linguagem Natural - PLN. O Sistema proposto foi avaliado com experimento com 20 redações, identificando e avaliando os erros de acordo com os erros já separados por corretores humanos. Os resultados obtidos demonstraram uma grande taxa de acerto a identificação e correção.
\end{abstract}

Palavras-Chave: PLN. Processamento de Linguagem Natural, avaliação de atividades escritas

\begin{abstract}
The use of information and communication technologies is increasingly standing out in Education. Currently, there are several technologies that provide support for distance learning. Before this scenario, there are millions of users with an huge amount of educational data, which evaluation burdens teachers and consume their time, leaving the students waiting for the evaluation of their performance. With the use of automatic correction technics performed by computers, there is a bigger fluidity of activities and a relevant decrease of work for the teachers, leaving them free to evaluate others activities. This paper presents a lexical-Syntactic Analyzer for automatic evaluation of written Activities in Portuguese using Genetic Algorithms and Natural Language Processing - NLP. The proposed system was evaluated by experiment, about 20 written activities and it found and evaluated the mistakes previously identified by human correctors. The results demonstrated a high rate of appropriate suggestions for the errors detected.
\end{abstract}

Keywords: NLP, Natural Language Processing, evaluation of written activities

DOI: 10.5753/RBIE.2016.24.92 


\section{Introdução}

Com o desenvolvimento da tecnologia, é cada vez mais comum o uso de Tecnologias Digitais da Informação e Comunicação - TDIC na educação, ajudando assim no ensino-aprendizagem [1]. Vários países vêm adaptando suas abordagens educacionais promovendo assim, cursos em diversas modalidades, sejam presenciais ou a distância. O Ensino Tradicional Professor-Aluno deixa de ser apenas uma modalidade presencial de ensino, e passa a ser ofertado, também, na modalidade de ensino a Distância, onde o aluno é um dos principais responsáveis por seu próprio aprendizado [2].

Com um novo olhar voltado para a educação, a EAD vem se tornando cada vez presente e ativa em ambientes presenciais de ensino-aprendizagem [3]. Assim, o uso de ambientes EAD é de fundamental importância para manter o contato professor-aluno fora da instituição física. No Ano de 2012, a Associação Brasileira de Educação à Distância - $\mathrm{ABED}^{6}$, divulgou que o número de usuários matriculados em ambientes EAD era de 5,8 milhões no Brasil, segundo o Censo EAD BR ${ }^{7}$. Em 2014 a ABED relatou que este número aumentou $52,5 \%$.

Atualmente, diferentes tipos de tecnologias educacionais apoiam a modalidade a distância, como por exemplo, os sistemas de gerenciamento de aprendizagem (LMS, do inglês Learning Management Systems) [4], Sistemas Tutores Inteligentes - STI [5], os sistemas educacionais de hipermídia adaptativa (AEHS, do inglês Adaptive Educational Hypermedia Systems) [6], os sistemas de aprendizagem colaborativa apoiados por computador (CSCL, do inglês Computer-Supported Collaborative Learning) [7] e, mais recentemente, os cursos online abertos e massivos (MOOCs, do inglês Massive Online Open Courses) [8]. Com isso, pode ser notado que com o passar do tempo, o ensino tradicional, Professor-Aluno, ganha novos rumos e estratégias de ensino.

Os sistemas de educação online são usados em diferentes níveis educacionais, seja no ensino fundamental, médio ou superior, e com diferentes propósitos, como auxiliar os alunos no desenvolvimento de provas e atividades [9]. Uma dessas provas é o ENEM (Exame Nacional do Ensino Médio), uma avaliação composta de questões de múltiplas escolhas e uma prova dissertativa, realizada nacionalmente pelo Ministério da Educação $\mathrm{MEC}^{8}$ utilizado para o ingresso de estudantes em redes de ensino superior. A quantidade de alunos realizando o

\footnotetext{
${ }^{6} \mathrm{http} / / / \mathrm{www} \cdot$ abed.org.br/site/pt/

${ }^{7}$ http://www.abed.org.br/site/pt/midiateca/censo_ead/1193/2013/09/

${ }^{8} \mathrm{http}: / /$ portal.mec.gov.br/
}

ENEM cresce anualmente (divulgação anual realizada pelo MEC da exame), como mostra a Figura 1, regis-

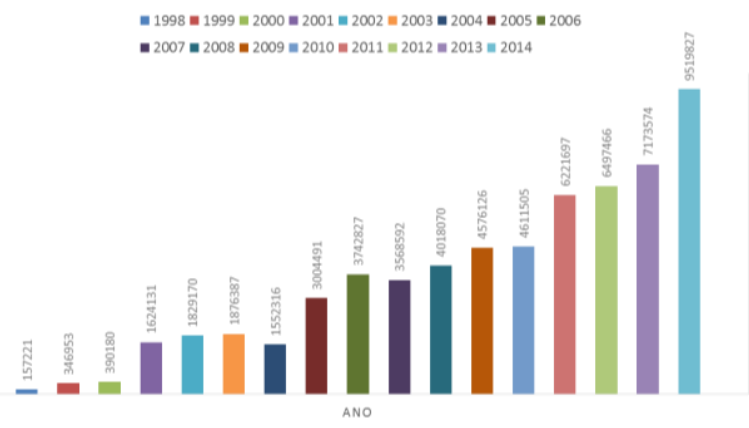

trando, em 2014, 9.519.827 inscrições.

Figura 1 : Aumento gradativo das inscrições para o ENEM entre os anos 1998-2014

Em 2014, o MEC publicou, como mostra a Figura 2, o balanço geral do ENEM, mostrando que a prova teve uma queda no desempenho dos estudantes, na avaliação escrita, de $9,7 \%$, sendo que aproximadamente $8,5 \%$, cerca de 530.000 estudantes obtiveram nota zero na

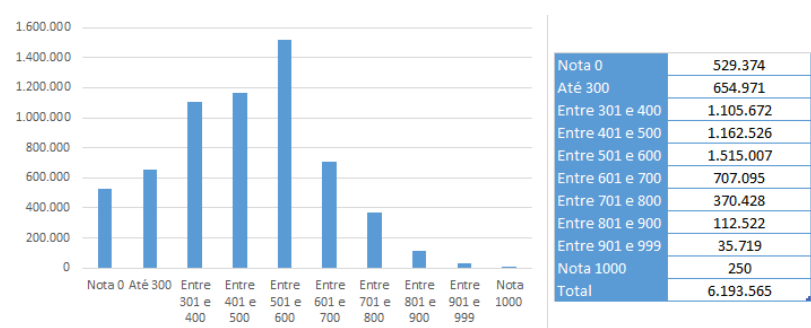

redação dos 6.193.565 alunos que realizaram a avalição.

Figura 2 : Balanço geral das notas da Redação - ENEM 2014 (Reprodução/INEP)

Diante do cenário de milhões de usuários, tem-se uma grande quantidade de dados disponíveis [10], principalmente ligada ao contexto de avaliações escritas, deixando o professor sobrecarregado [11] para avaliar esses dados individualmente e ao aluno, fica a responsabilidade de aprender de forma autônoma [12]. Com isso, há uma necessidade de personalização da aprendizagem que auxilie o aluno [13], não somente avaliando e dando a resposta correta, mas sim, identificando o erro e fornecendo sugestão de correção e melhoria contínua na sua formação.

É fundamental o uso de TIC com capacidade de processar estes dados de forma a atender as necessidades individuais de cada aluno, ligadas às dúvidas que os 
usuários podem ter quanto ao exercício que está sendo realizado, fazendo com que tal sobrecarga não influencie negativamente a interação Professor-Aluno [11].

Para tanto, sabendo que atividades escritas em ambientes de educação online, quando avaliadas, geram sobrecarga por parte do professor, a literatura utiliza técnicas de Processamento de Linguagem Natural (PLN) nesses ambientes de educação online para a automação da análise ortográfica e gramatical das atividades escritas. Para atender as necessidades dos alunos por espera de um feedback, se fez uso de Algoritmos Genéticos, auxiliando no processo de correções, oferecendo as sugestões ótimas para os erros.

O principal objetivo deste trabalho é apresentar um Sistema Ortográfico e Gramatical que possa ser incorporado em ambientes de aprendizagem online de forma que amenize o trabalho do professor ou tutor responsável por corrigir atividades escritas. A aplicação passou por um experimento, seguindo as competências do ENEM ligadas ao fator léxico ortográfico e gramatical, no que se refere a demonstrar domínio da modalidade escrita formal da Língua Portuguesa, tratando e verificando palavras escritas de forma incorreta até sentenças com problemas relacionados desde o uso da crase até as concordâncias, tendo resultados semelhantes ao de um especialista, nesse contexto, professor. O trabalho visa contribuir com a avaliação de textos escritos no contexto de ambientes de aprendizagem a distância, fazendo uso de corretores gramaticais e ortográficos, desde aspectos ligados às estruturas das palavras, uso de dicionários, até fatores que influenciam o contexto da estrutura da frase.

Este trabalho estende os resultados presentes no artigo publicado em [14], apresentando um melhor emba- samento teórico, facilitando o entendimento de técnicas utilizadas para o processamento de linguagem natural, para um entendimento de todo o processo desenvolvido e quais técnicas foram utilizadas e com quais propósitos nesse artigo. As técnicas possuem um nível de detalhe, explicando como e onde elas foram implementadas, e o que se pretendeu alcançar com tal implementação. Os resultados foram discutidos para a diminuição da sobrecarga por parte do professor, incrementando a análise tanto funcional do sistema quanto de uma comparação com os resultados de um especialista, destacando detalhadamente a semelhança das correções tanto realizadas por um especialista quanto pelo sistema proposto.

\section{Fundamentação Teórica}

O objetivo desta seção é apresentar conceitos importantes que auxiliam na compreensão da pesquisa desenvolvida na área da linguística aplicada na computação.

\subsection{Definições e técnicas de Processamento de Linguagem Natural}

Termo Léxico é o conjunto de palavras de um determinado idioma [15]. Termo Sintático é o conjunto de relações existentes entre palavras de uma oração ou de orações em períodos distintos [16].

A POS Tagging ou etiquetagem é a marcação de uma classe gramatical [17]. Ela é responsável pela identificação dos itens lexicais e da classe que determinada palavra, em uma sentença, possui, gerando uma árvore de derivação sintática, como mostra a Figura 3. A principal função desempenhada pela etiquetagem é a resolução das ambiguidades.

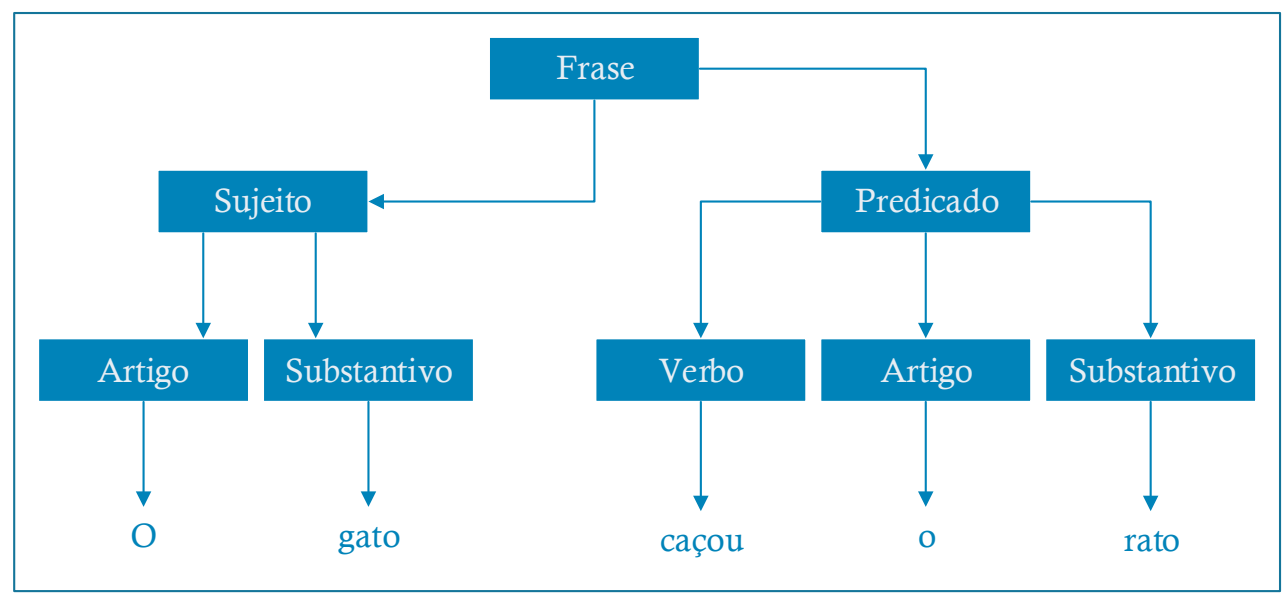

Figura 3 : Árvore de derivação sintática 
Os etiquetadores podem ser construídos (i) baseados em regras [18], restrições [19], casos [20] e em árvores de decisões não probabilísticas, denominados simbólicos, (ii) baseados em modelos probabilísticos [21] podendo utilizar de redes neurais [22], máxima entropia [23], Modelo de Markov [24] e árvores de decisão probabilísticas [21] para calcular qual a probabilidade de determinada palavra receber a etiqueta com seu respectivo contexto, denominado estocásticos, ou (iii) híbridos, que são baseados na combinação dos dois modelos apresentados anteriormente, ou seja, o processo de etiquetagem deste modelo emprega tanto os modelos baseados em regras quanto os modelos estocásticos. Um modelo bastante conhecido na literatura que utiliza esse tipo de abordagem é o etiquetador baseado em transformações - TBL [25], [26], [27]. Logo abaixo, são exibidas as etiquetas que geralmente são atribuídas pelos etiquetadores:

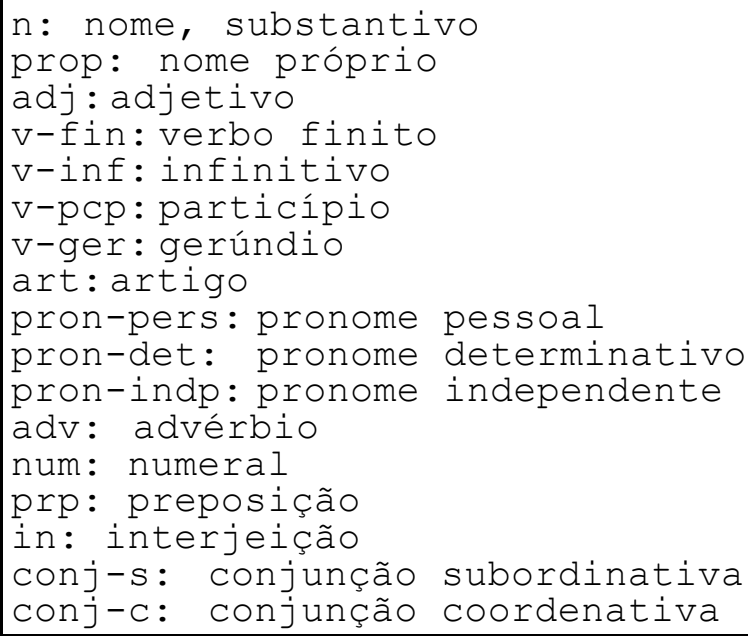

Denomina-se analisadores sintáticos (em inglês, parsers) como os sistemas que realizam a análise estrutural e seus constituintes. Os parsers são responsáveis pelo reconhecimento de estruturas válidas a partir de um termo léxico cuja responsabilidade é definir o vocabulário e o conjunto de regras, compondo assim, a gramática da língua [28]. O analisador léxico é usado pelo sintático a fim de reunir os itens lexicais da língua e de sua respectiva gramática [29].

\subsection{Algoritmo Genético}

Algoritmos Genéticos (AGs) são modelos computacionais que utilizam técnicas inspiradas na evolução biológica (seleção natural) [30]. São destinados para encontrar uma solução otimizada, ou melhor, uma solução potencial para determinado problema [31]. Em AGs, existem alguns termos que são comumente usados, como cromossomo que são os elementos constituintes do indi- víduo, indivíduo são os que formam a população.

\subsubsection{Funcionamento de um Algoritmo Gené- tico simples}

Um Algoritmo Genético conta com uma inicialização, onde basicamente é criada uma população inicial, logo em seguida sucedem algumas etapas como avaliação, seleção, cruzamento, mutação, atualização e a finalização [30], conforme Figura 4.

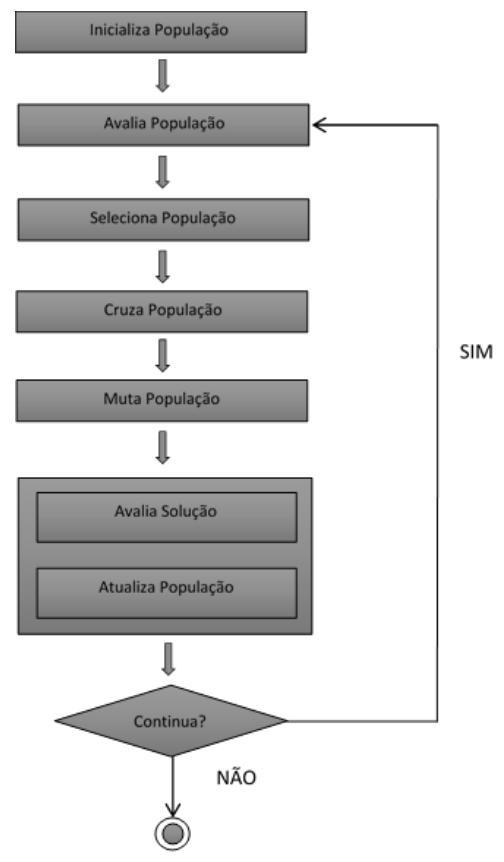

Figura 4 : Processo de avaliação em um algoritmo genético [30]

$\mathrm{Na}$ avaliação, verifica-se e valida à aptidão das soluções, que são basicamente os indivíduos da população. É realizada uma análise para verificar o quão próximo da solução do problema a população se encontra [30].

Na seleção, os indivíduos são selecionados para a reprodução. Para validar esta etapa, verifica-se uma probabilidade; dado uma solução i, a probabilidade de ser selecionada é proporcional à sua aptidão [30].

No cruzamento ou reprodução, as características dos indivíduos são recombinadas, formando um novo grupo de indivíduos [30]. Na mutação, as características dos indivíduos gerados na etapa anterior são modificadas, formando assim uma nova característica à população [30]. Na atualização, os indivíduos gerados nas etapas anteriores, são inseridos na população [30].

Por fim, a finalização, responsável por verificar as condições de encerramento, determinando se determinada população atingiu uma solução ótima, caso contrário, todo o processo anterior é executado novamente. 


\subsection{Algoritmo Levenshtein Distance}

É uma métrica de avaliação da similaridade entre duas palavras. Esse algoritmo foi desenvolvido pelo cientista russo Vladimir Levenshtein em 1965 [36]. Tem como base realizar comparações para tentar transformar uma palavra em outra, avaliando a substituição, inserção e exclusão dos caracteres. A complexidade computacional do algoritmo Levenshtein Distance - LD vem sendo estudada ao longo do tempo e otimizações em relação ao desempenho computacional são realizadas [32].

Aproximadamente $80 \%$ das palavras digitadas de forma incorreta possuem pelo menos um erro com relação à inserção, remoção ou substituição dos termos [33]. Quando a distância é igual à zero, indica que as palavras são idênticas. Uma matriz é montada contendo os custos de cada operação. Após as comparações, tem-se a distância ocupando a última posição da matriz.

\section{Motivação}

Existe uma carência para avaliar atividades dissertativas/discursivas em ambientes de Educação Online, visto que em um contexto de milhões de usuários, tal tarefa apresenta uma sobrecarga para professores e tutores, tornando-se cada vez mais inviável a correção da produção textual de forma personalizada devido à quantidade de estudantes presentes nesses ambientes.

Com isso, nota-se a necessidade de implantação de soluções que diminuam a sobrecarga nos professores e tutores, dando suporte à realização de atividades escritas em ambientes de Educação Online.

\section{Proposta}

Esta seção trata sobre a proposta de criação de uma sistema para avaliação automática de atividades escritas. Nela é apresentado tanto a criação dos módulos ortográfico e gramatical, que constituem a ferramenta, quanto a junção destes em um único módulo, mostrando a relação que cada um tem com o outro.

O sistema tem como objetivo reduzir a sobrecarga de professores e tutores, no que tange a avaliação de atividades escritas. Faz-se isso destacando os erros e oferecendo, aos próprios alunos, sugestões de correção ortográfica e/ou sintática, auxiliando-os no processo de aprendizagem.

\subsection{Classificador}

Este componente realiza a classificação gramatical das palavras, utilizando etiquetagem, gerando uma árvore de derivação [34]. Para palavras onde a classe grama- tical correspondente não é encontrada, o classificador marca a Tag neutra, indicando que não foi possível encontrar a classe gramatical correspondente. Na Figura 5, são apresentadas duas frases demonstrando o processo de classificação.

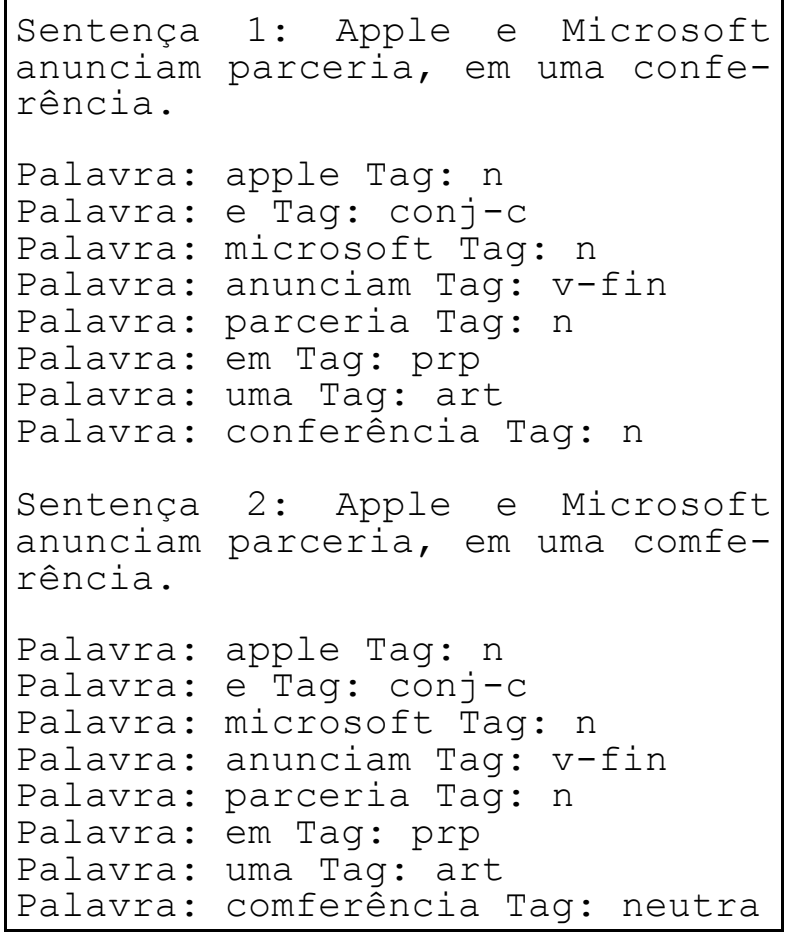

Figura 5: Correção realizada pelo Módulo ortográfico - Identificação e sugestões de palavras

Como pode ser observado, o classificador não conseguiu encontrar a classe correspondente à palavra "comferência". Com isso, a palavra é marcada pela Tag neutra, indicando que não é possível efetuar uma classificação exata. Tal método de classificação é fator chave para o módulo ortográfico, que será apresentado a seguir.

\subsection{Módulo Ortográfico}

O Módulo Ortográfico é responsável por realizar todo o tratamento de verificação ortográfica das palavras, sugerindo correções quando um erro é detectado.

O módulo ortográfico foi criado com o intuito de realizar a correção léxica das palavras, ou seja, encontrar palavras escritas de forma errada e sugerir palavras para a substituição desta, conforme a Figura 6 . O objetivo é impedir que palavras grafadas incorretamente impossibilitem, ou interfiram, no processo de correção sintática (feita pelo módulo gramatical). No módulo ortográfico, encontra-se o dicionário Auxiliar, que é responsável por realizar a correção ortográfica das palavras que não estão 
presentes no dicionário principal, chamado JSpell ${ }^{1}$. No dicionário Auxiliar, ao contrário do JSpell, é possível adicionar novas palavras, corretas, que foram identificadas no texto. O motivo da criação de um dicionário auxiliar foram as dificuldades encontradas no momento da inserção de novas palavras e ao acesso, por parte do módulo ortográfico, ao JSpell.

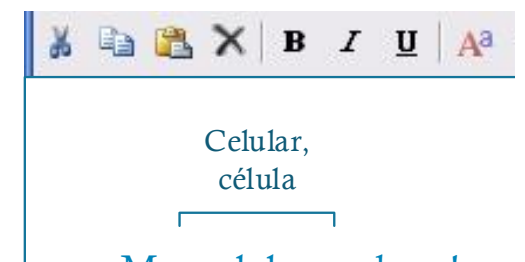

Meu selular quebrou!

Figura 6 : Correção realizada pelo Módulo ortográfico - Identificação e sugestões de palavras

O módulo ortográfico utiliza técnicas de PLN (Processamento de Linguagem Natural), como o algoritmo Levenshtein Distance, para otimizar as sugestões de correção feitas pelo Algoritmo Genético. Dado a presença de um erro ortográfico no texto, o algoritmo genético utilizado para estimar as sugestões das palavras, possui o algoritmo Levenshtein Distance para ordenar, por similaridade, as palavras selecionadas e retornar para o usuário. A Figura 7 detalha melhor o processamento realizado nesta etapa.

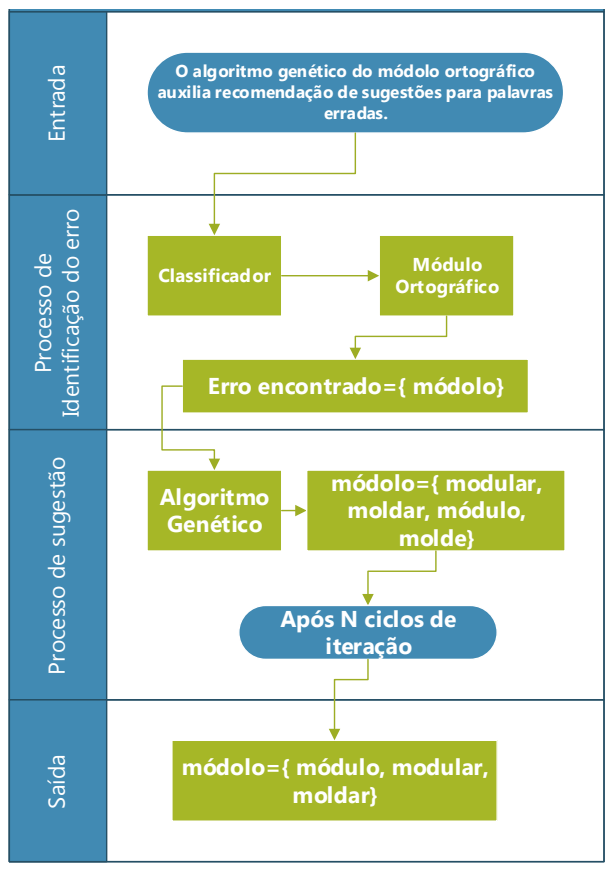

Figura 7 : Processo de correção ortográfica

\subsection{Módulo Gramatical}

O Módulo Gramatical é o componente responsável por realizar todo o tratamento de verificação gramatical do texto, efetuando as devidas sugestões gramaticais para a solução de um erro detectado.

No Módulo Gramatical, encontra-se o dicionário JSpell, que atua como dicionário principal do sistema. Utilizando sistemas com código aberto, como o CoGrOO [35], o módulo gramatical foi gerado, adaptado e organizado em uma nova arquitetura que pudesse ser acoplado ao módulo ortográfico, como pode ser observado na Figura 7.

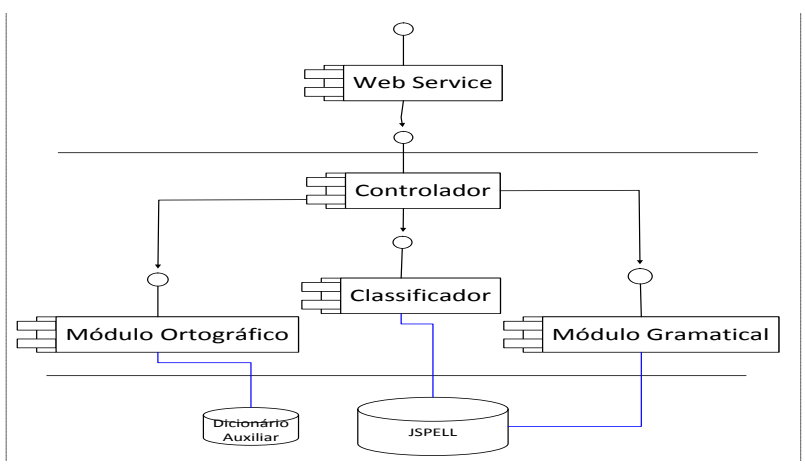

Figura 7 : Arquitetura do Sistema

Com a organização da nova arquitetura, um controlador, componente responsável por sincronizar as funcionalidades dos componentes classificador e módulo ortográfico, foi criado para facilitar a comunicação entre as camadas de serviço e kernel. Com o controlador, é possível separar os módulos e executar a atividade de acordo com a demanda, assim, caso não fosse necessário executar uma varredura para encontrar erros ortográficos, processo ativo com a presença da Tag neutra, a performance teve melhorias consideráveis.

O módulo gramatical reconhece erros de concordância verbal, concordância nominal, uso de crase e colocação pronominal, entre outros, como pode ser observado na Figura 8.

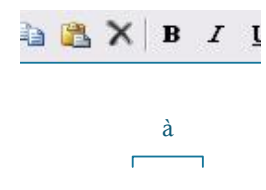

Eu vou a praia

Figura 8 : Identificação e sugestões de palavras

${ }^{1}$ https://www.jspell.com/ 
Os erros são identificados e mapeados em TAG (etiquetas) contendo informações relacionadas às palavras, tais como: questão de gênero, número, classe gramatical, através de Tokens.

O analisador gramatical utiliza técnicas de PLN, como POS Tagging, apresentada na seção 2.3 , recebendo a redação e logo em seguida executa a correção. Todas as regras gramaticais foram mapeadas em XML.

\subsection{Funcionamento}

Inicialmente, uma questão dissertativa (texto) é submetida pelo aluno. A questão é, então, analisada pelo classificador que realiza uma classificação das palavras em suas respectivas classes gramaticais, todas contidas em uma lista. Toda a classificação acontece com o uso do dicionário principal JSpell.
Para as palavras que não forem encontradas uma classificação gramatical correspondente, o analisador marca a palavra com uma TAG específica, denominada Neutro. Esta marcação indica que o componente não consegue analisar a palavra, entre outras palavras, a palavra analisada não possui classe gramatical, e que o analisador ortográfico será o responsável pela análise de tal palavra. Depois de executar todas as classificações e marcações das palavras, quando for necessário, a etapa de avaliação ortográfica-gramatical é iniciada.

As sentenças são avaliadas de acordo com esses Tokens que estão contidos nas listas. Foram utilizadas técnicas disponíveis no Apache OpenNLP ${ }^{1}$. A correção é realizada com sugestões de acordo para cada tipo de erro que foi encontrado, o corretor recebe um texto, avalia e retorna as devidas sugestões para as palavras ou expressões que forem identificadas como erradas, conforme a Figura 9.

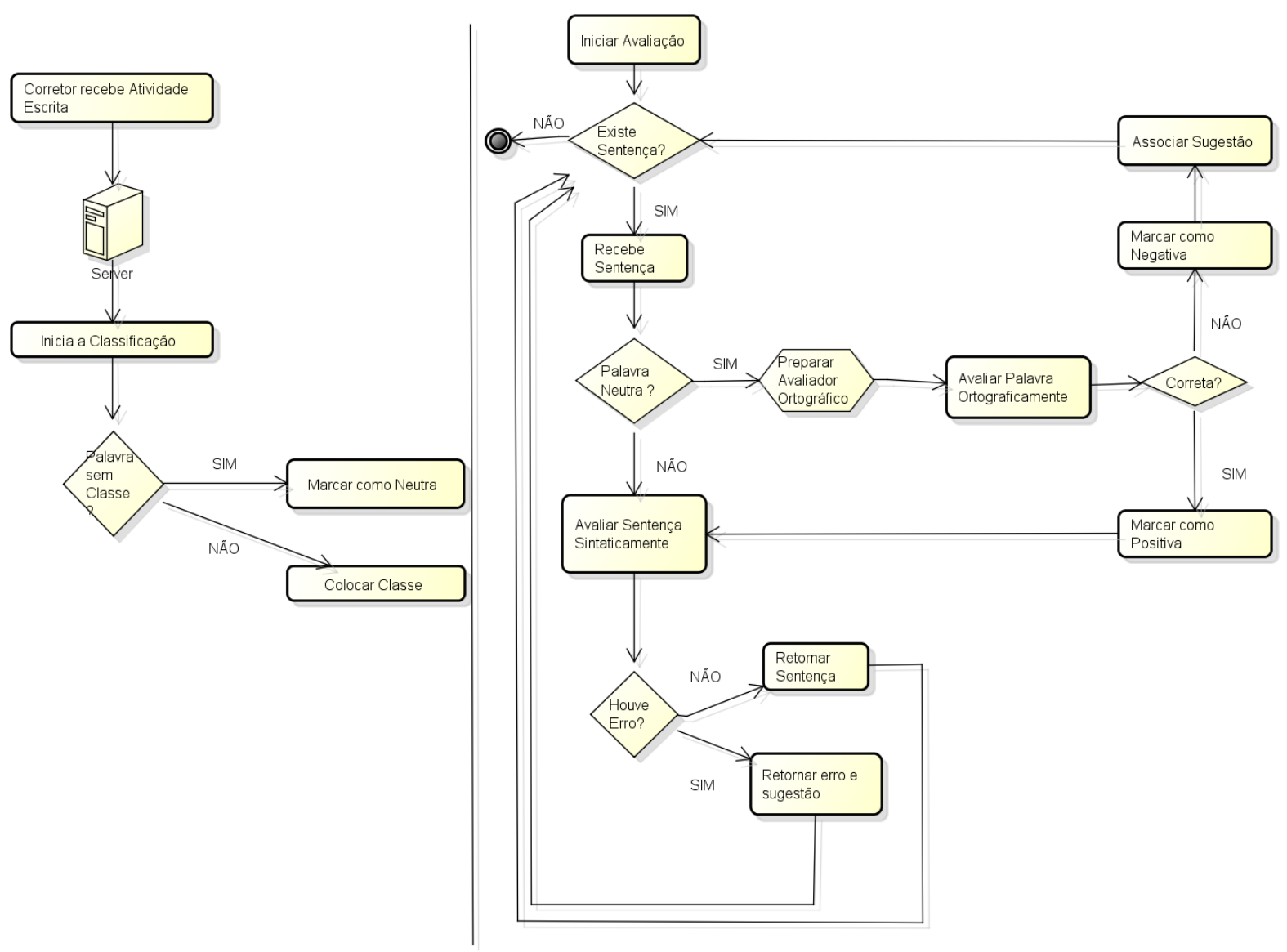

Figura 9 : Fluxo de avaliação de uma redação - Sentença por Sentença

${ }^{1}$ https://opennlp.apache.org/ 
Quando o avaliador gramatical encontra uma palavra com marcação Neutra, repassa para o avaliador ortográfico, onde, são avaliadas e posteriormente, marcadas com uma das seguintes TAGs:

(i) Positivas: São as palavras Neutras que foram consideradas corretas depois da avaliação do módulo ortográfico;

(ii) Negativas: São as palavras Neutras que foram consideradas como erradas logo após a validação pelo módulo ortográfico.

Para remarcar as palavras Neutras como Positivas ou Negativas, o analisador ortográfico consulta o dicionário auxiliar, verificando a presença ou não da palavra. Quando a palavra é encontrada, o analisador substitui a marcação Neutra pela marcação Positiva, indicando que a palavra existe e está grafada corretamente. Quando a palavra está ausente, ou está escrita incorretamente, ela é marcada como Negativa.

Considerando as palavras marcadas como Negativas, o avaliador ortográfico utiliza técnicas de algoritmos genéticos para recomendar possíveis correções. Para isso é criada uma população inicial com base em alterações de caracteres da palavra. Após verificações como quantidades de caracteres com a palavra que se quer encontrar, algumas funções começam a induzir palavras que se assemelham à palavra errada, utilizando consultas com caracteres especiais SQL e, logo após, o algoritmo repassa toda a solução gerada de palavras para a função baseada no algoritmo Levenshtein Distance [36]. As melhores sugestões são vinculadas à palavra que já está com a marcação Negativa e são retornadas para o avaliador gramatical continuar sua avaliação.

Como pode ser percebido, a técnica de correção ortográfica ocorre durante a validação gramatical das palavras, o que de certa forma, influencia e colabora com o módulo ortográfico, pois no momento da avaliação gramatical, o Módulo Gramatical já age marcando as palavras em prováveis erros, o que facilita a identificação e onde pode ser encontrado tal erro, passando esta informação dinamicamente para o módulo ortográfico, que avalia e dá a classificação como um erro e sua respectiva sugestão, ou como uma palavra correta.

O algoritmo genético faz a população inicial com base em um dicionário. Algumas funções fitness validam a população criando novas populações de palavras que melhor se adequam à palavra incorreta. Em uma dessas funções de fitness foi aplicado o algoritmo LD - Levenshtein Distance [36]. A técnica calcula e aproxima quais palavras estão mais próximas da palavra incorreta.

Com base nos valores gerados pelo cálculo da distância entre as palavras sugeridas, uma nova função fitness avalia e ordena os valores, colocando as palavras que obtiveram a menor distância gerada pelo algoritmo LD [36]. As mutações são realizadas com base nas palavras que obtiveram o menor cálculo da distância, retirando caracteres e acrescentando novos com base em consultas ao dicionário. A reprodução se dá através da combinação das palavras que mais se aproximaram da palavra incorreta, verificando e validando através do dicionário. Então, uma sugestão é feita para o analisador Gramatical que novamente valida a sentença e a repassa para o usuário. A Figura 10 apresenta um pseudo-algoritmo mostrando o processo de avaliação de uma sentença. 


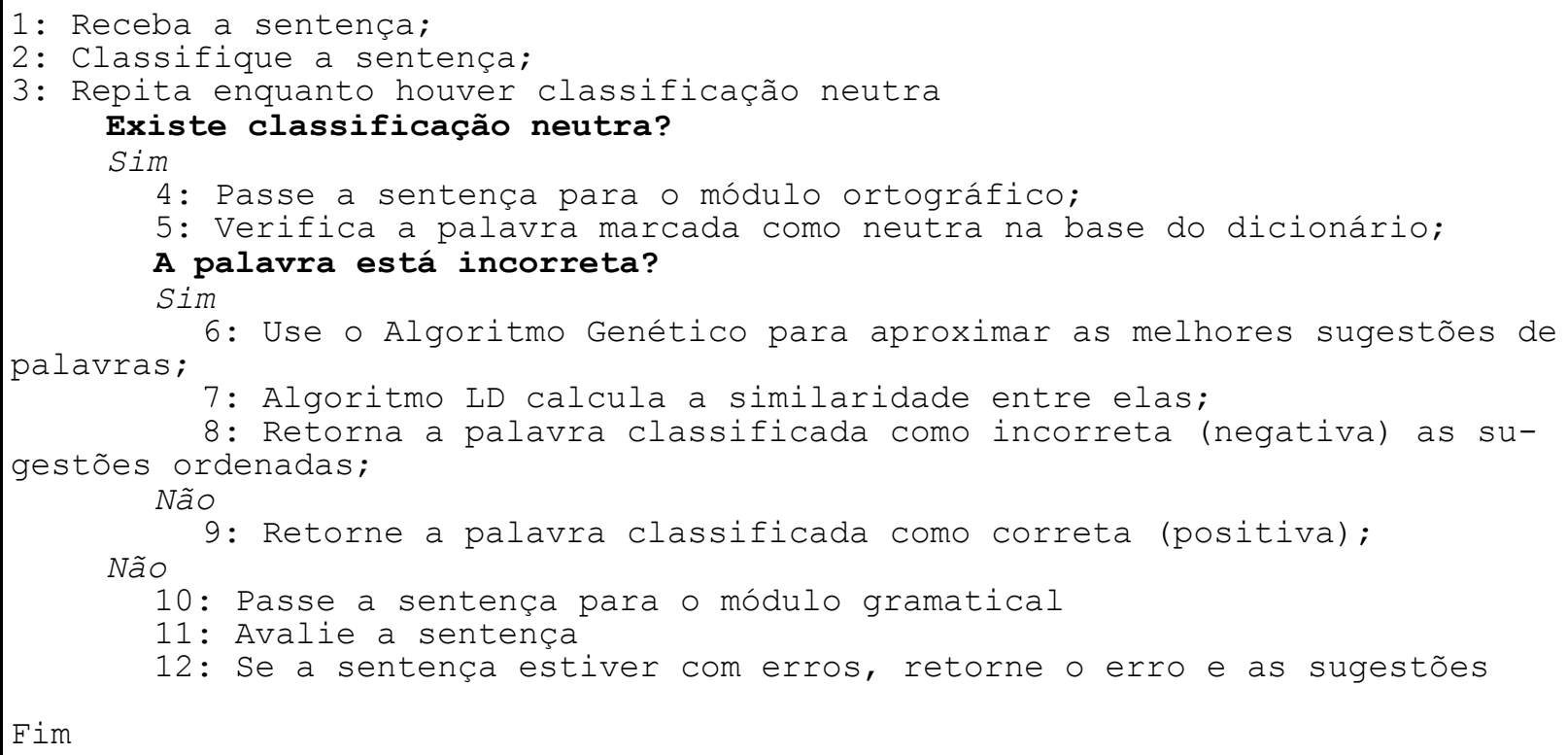

Figura 10 : Pseudo-código representando a avaliação de uma sentença

Os passos 1 e 2 do Pseudo-código fazem parte da avaliação realizada pelo Classificador. Contudo, para todas as vezes que existir uma palavra sem uma determinada classe gramatical, será necessário verificar esta palavra pelo Módulo Ortográfico, uma vez que por não possuir uma classe correspondente, pode indicar que esta palavra é um provável erro, passo 3. Com isso, os Passos 4, 5, 6, 7,8 e 9 são desempenhados pelo Módulo Ortográfico. Para tanto, os passos 10, 11 e 12 são executados pelo Módulo Gramatical.

\section{Experimento}

Nesta seção, apresenta-se um experimento para o levantamento e análise funcionais do Módulo Ortográfico - Gramatical, tanto em questões ligadas à execução destes de forma separada, quanto em funcionamento acoplado.

O objetivo do experimento foi diminuir a sobrecarga por parte de professores/tutores e avaliar a estrutura ortográfica e sintática de Redações do ENEM, oferecendo sugestões e observar o funcionamento dos módulos tanto de forma isolada quanto integrada, encontrar limitações e erros para possíveis melhorias.

\subsection{Planejamento do Experimento}

No experimento, serão comparadas as correções realizadas tanto por parte do sistema corretor quanto do especialista. $\mathrm{O}$ contexto do experimento é científico.

A criação de um Sistema Corretor Ortográfico-
Gramatical nos levou aos seguintes questionamentos:

- Como podemos avaliar a qualidade das correções realizadas pelo sistema corretor?

- Tais correções podem ser comparadas às correções realizadas por um especialista (professor/tutor)?

- Será que tal modelo de correção automática contribui na redução da carga sobre o professor?

Para tais questionamentos, podemos destacar algumas métricas que poderiam nos levar às respostas dessas perguntas, como por exemplo:

- Semelhança entre a quantidade dos erros encontrados ortograficamente e sintaticamente tanto do sistema corretor quanto do especialista no critério de Precision

- Semelhança entre a quantidade dos erros encontrados ortograficamente e sintaticamente tanto do sistema corretor quanto do especialista no critério de Recall

Os resultados das execuções serão analisados sob possíveis métricas de custo, redução da sobrecarga no professor/tutor e a eficácia das correções com a implantação do sistema corretor. Para avaliar a eficácia será realizada uma comparação entre os resultados obtidos pelo sistema corretor e os resultados obtidos por especialistas. Formalmente, o objetivo da investigação pode ser definido no formato GQM como: analisar a qualidade de 
correção do sistema corretor proposto com a intenção de avaliá-lo a respeito de seu custo, benefícios aos professores/alunos, sob ponto de vista dos usuários de ambientes educacionais no contexto dos ambientes EAD com atividades escritas.

O Plano de execução do experimento é:

(i) Selecionar redações contendo erros ortográficos e sintáticos sem restrições de temas

(ii) Selecionar um mediador para fazer o intermédio do experimento, com o objetivo de garantir confiabilidade, e fluidez do experimento.

(iii) Preparar o ambiente, visando a detecção de erros ortográficos e sintáticos.

(iv) Executar o experimento

(v) Coletar os dados

(vi) Fazer a análise de tais dados

(vii) Discutir os resultados

\subsection{Execução}

O experimento foi realizado com 20 redações disponíveis na $\mathrm{WEB}^{11}$, utilizando o novo Acordo Ortográfico da Língua Portuguesa. As redações seguem o modelo de escrita para o Exame Nacional do Ensino Médio com suas devidas correções realizadas por professores/tutores.

Para avaliações escritas, o INEP ${ }^{12}$ designou algumas competências para a avaliação da redação presente no ENEM. As competências foram divididas em cinco módulos:

(i) Demonstrar domínio da norma padrão da língua escrita.

(ii) Compreender a proposta de redação e aplicar conceitos das várias áreas de conhecimento para desenvolver o tema, dentro dos limites estruturais do texto dissertativo-argumentativo.

(iii) Selecionar, relacionar, organizar e interpretar informações, fatos, opiniões e argumentos em defesa de um ponto de vista.

(iv) Demonstrar conhecimento dos mecanismos linguísticos necessários para a constru-

\footnotetext{
11 http://educacao.uol.com.br/bancoderedacoes/ - Banco de Redações disponibilizadas na WEB

${ }^{12}$ INEP: Instituto Nacional de Estudos e Pesquisas

${ }^{13} \mathrm{http}$ ://www.inep.gov.br/
}

ção da argumentação.

(v) Elaborar proposta de solução para o problema abordado, respeitando os valores humanos e considerando a diversidade sociocultural

As redações foram escolhidas com base em erros ortográficos e sintáticos, pois o intuito do experimento foi avaliar a primeira competência do ENEM: Demonstrar Domínio da norma padrão da língua escrita sob os critérios ortográficos e sintáticos. Todas contendo uma quantidade mínima de 300 caracteres. A partir desta seleção, as correções realizadas pelos professores/tutores foram removidas, mapeadas em uma planilha para efetuar a comparação com as correções realizadas pelo sistema. A partir disso, as redações foram divididas em cinco categorias para cada módulo distinto de acordo com a quantidade de erros sintáticos e ortográficos presentes.

Para a divisão das categorias avaliadas no módulo ortográfico, temos:

(i) Categoria 1: Possuía entre 1 e 13 erros ortográficos;

(ii) Categoria 2: Possuía entre 14 e 20 erros ortográficos;

(iii) Categoria 3: Possuía entre 21 e 30 erros ortográficos;

(iv) Categoria 4: Possuía entre 30 e 35 erros ortográficos;

(v) Categoria 5: Possuía entre 35 e 40 erros ortográficos;

Para a divisão das categorias avaliadas no módulo gramatical, temos:

(i) Categoria 1: Possuía entre 1 e 3 erros gramaticais;

(ii) Categoria 2: Possuía entre 4 e 7 erros gramaticais;

(iii) Categoria 3: Possuía entre 8 e 9 erros gramaticais;

(iv) Categoria 4: Possuía entre 7 e 9 erros gramaticais seguidos de erros ortográficos;

(v) Categoria 5: Possuía entre 9 e 13 erros gramaticais seguidos de erros ortográficos;

As categorias foram classificadas em duas classes distintas para cada módulo. A classe 1 de ambos os módulos tinha o objetivo de avaliar o comportamento inicial do sistema, verificando a quantidade de erros encontra- 
dos e avaliados com os erros que deveriam ser encontrados, recall e precision, respectivamente. Já, a Classe 2 tinha o objetivo de avaliar o sistema cumprindo os requisitos de avaliador ortográfico-gramatical, seguindo a competência do Enem, bem como todo o processo de avaliação, desde o reconhecimento dos erros até suas devidas correções e sugestões.

As Classes do Módulo Ortográfico eram:

(i) Classe 1: Composta de neologismos, Nomes Próprios, Siglas, Datas e prováveis símbolos, como o “@” do e-mail, e alguns erros ortográficos;

(ii) Classe 2: Composta de erros ortográficos.

\section{As Classes do Módulo Gramatical eram:}

(i) Classe 1: Composta por erros sintáticos;

(ii) Classe 2: Composta tanto por erros sintáticos quanto erros ortográficos;

\subsection{Métricas}

A avaliação das redações ocorreu com os módulos isoladamente e nos módulos em funcionamento em conjunto. Os critérios para a avaliação do sistema foram Precisão e Recall, dados pelas equações (1) e (2) respectivamente.

$$
\begin{aligned}
& p=\frac{(t p)}{(t p+f p)} \\
& r=\frac{(t p)}{(t p+f n)}
\end{aligned}
$$

- $\operatorname{tp}=$ verdadeiros positivos

- $\mathrm{fp}=$ falsos positivos

- $\mathrm{fn}=$ falsos negativos

\section{Resultados e discussão}

Nesta seção, se encontram os resultados decorrentes da análise (capítulo 5). Os resultados foram divididos em classes, relacionando cada módulo com um conjunto específico, avaliando-os em funcionamento separado e em funcionamento em conjunto.

\subsection{Módulo Ortográfico}

A avaliação foi dividida de acordo com as classes se- paradas das redações. Dois testes foram realizados mostrando o nível de exatidão não somente comparando os erros que o módulo deveria encontrar, mas também com as sugestões de palavras que ele deveria fazer.

\subsubsection{Avaliação do resultado para classe 1}

O algoritmo cumpriu a primeira etapa com $60 \%$ (total de redações sem correção errada) das redações avaliadas com sucesso, como mostra a Tabela 1 e a Figura 11. Os $40 \%$ que tiveram quantidade de erros identificados, diferente da quantidade de erros existentes. Isso se deu pelo fato de que novas palavras, que não estavam mapeadas no dicionário, não puderam ser avaliadas nem identificadas. Contudo, o analisador ortográfico conseguiu dar sugestões de palavras que se assemelhavam ao que

\begin{tabular}{|c|c|c|}
\hline Redações & Erros & Correções \\
\hline R1 & 5 & 5 \\
\hline R2 & 8 & 8 \\
\hline R3 & 8 & 9 \\
\hline R4 & 12 & 12 \\
\hline R5 & 14 & 15 \\
\hline
\end{tabular}
estaria sendo considerado erro.

Tabela 1: Resultados classe 1 - Módulo Ortográfico

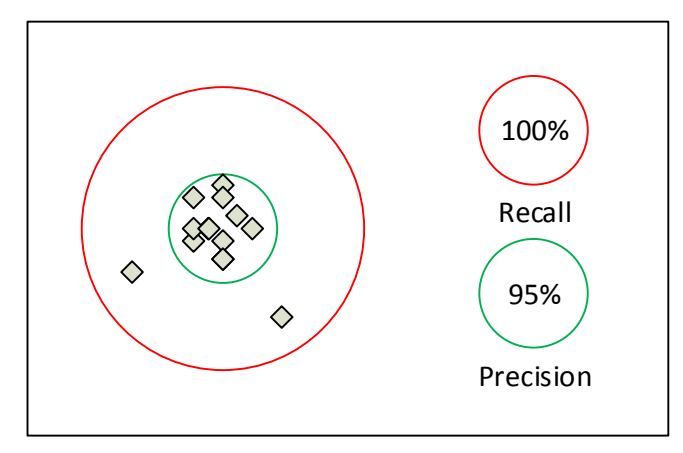

Figura 11 : Precision e Recall para Classe 1 - Módulo Ortográfico

\subsubsection{Avaliação do resultado para classe 2}

A avaliação dos resultados da classe 2 foi de $80 \%$ de acertos relacionados à quantidade de erros esperados, exibidos na Tabela 2 e Figura 12 . Os $20 \%$ foram avaliados e todos os erros foram encontrados com 3 palavras que foram classificadas como erros sem serem erros, pois

\begin{tabular}{|c|c|c|}
\hline$S \quad$ Redaçõe & Erros & Correções \\
\hline R6 & 16 & 16 \\
\hline R7 & 18 & 18 \\
\hline R8 & 17 & 20 \\
\hline R9 & 15 & 15 \\
\hline
\end{tabular}
não constavam na base de dados do dicionário. 


\begin{tabular}{l|ll}
\hline $\mathbf{R} 10$ & 21 & 21 \\
\hline
\end{tabular}

Tabela 2: Resultados classe 2 - Módulo Ortográfico

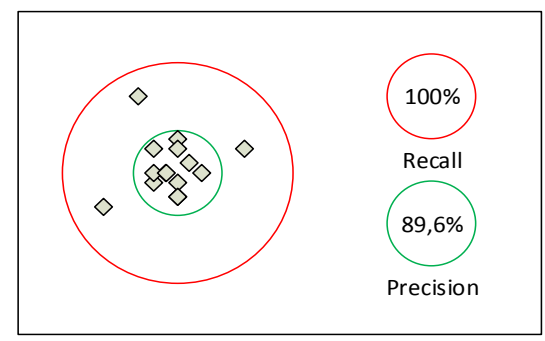

Figura 12 : Precision e Recall para Classe 2 - Módulo Ortográfico

\subsubsection{Avaliação do resultado geral}

O analisador Ortográfico se comportou de maneira esperada, visto que o não reconhecimento de palavras novas seria a principal forma de classificação de palavras como verdadeiros negativos, palavras classificadas como erros sendo que não eram erros, apresentado no Gráfico 2.

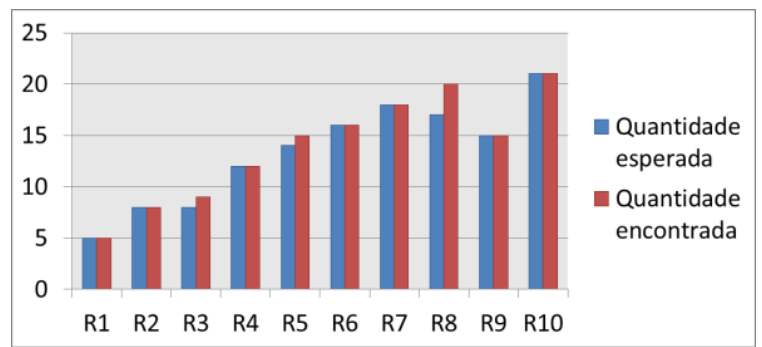

Gráfico 2 : Resultado geral correção ortográfica

Tendo em vista um Total de 134 erros ortográficos que deveriam ser encontrados, o Módulo Ortográfico encontrou 139 erros, dos quais, esses erros eram palavras que não estavam presente na base do dicionário, sendo assim, 5 palavras desse resultado podem ser classificadas como falso positivo, como mostra o Gráfico 3 .

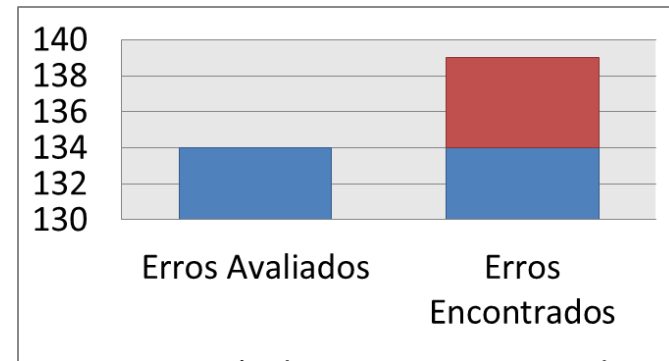

Erros Avaliados $\square$ Erros Encontrados

Gráfico 3 : Resultado geral quantidade de erros encontrada

\subsection{Módulo Gramatical}

A avaliação seguiu o mesmo padrão da avaliação executada no módulo ortográfico.

A classe 1 para a análise Gramatical era formada de expressões que somente possuíam erros gramaticais. As sentenças das redações possuíam erros que envolviam uso da crase, concordância nominal e verbal, entre outros. Abaixo, pode-se encontrar, na Tabela 3 e Figura 13, a quantidade de erros que estava presente em cada redação.

\begin{tabular}{|c|c|c|}
\hline Redações & Erros & Correções \\
\hline R11 & 1 & 1 \\
\hline R12 & 2 & 2 \\
\hline R13 & 4 & 3 \\
\hline R14 & 3 & 3 \\
\hline R15 & 6 & 4 \\
\hline
\end{tabular}

Tabela 3: Resultados classe 1 - Módulo Gramatical

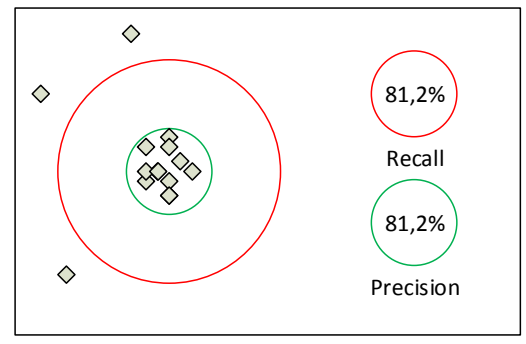

Figura 13 : Precision e Recall para Classe 1 - Módulo Gramatical

O analisador gramatical agiu com $60 \%$ de acertos, não conseguindo encontrar 3 erros. Por ter sido modificado para receber o módulo ortográfico, algumas alterações quanto ao uso da crase e concordância verbal.

$\mathrm{Na}$ classe 2, erros ligados à classe 1 e erros voltados para a ortografia também estavam presentes. As correções são mostradas na Tabela 4 e Figura 14. Teve-se uma taxa de $60 \%$ com base nos erros esperados. O que mais chamou a atenção foi que os erros ortográficos influenciaram na validação Gramatical. O Módulo Gramatical não conseguiu identificar alguns erros por conta da posição onde determinados erros ortográficos se encontravam. A sentença "(...). Elez deve se casar (...)" possui um erro ortográfico localizado logo após um ponto. Como se inicia uma palavra com letra maiúscula após um ponto, o analisador gramatical verificava e considerava "Elez" como um nome próprio, tendo uma ambiguidade, com isso, não poderia induzir uma correção gramatical quanto à sentença. 


\begin{tabular}{|c|c|c|c|c|c|}
\hline Redações & $\begin{array}{l}\text { Erros } \\
\text { Gram. }\end{array}$ & $\begin{array}{c}\text { Erros } \\
\text { Ort. }\end{array}$ & $\begin{array}{l}\text { Correções } \\
\text { gramaticais }\end{array}$ & $\begin{array}{l}\text { Correções } \\
\text { sintáticas }\end{array}$ & Total \\
\hline R16 & 3 & 2 & 3 & 0 & 3 \\
\hline R17 & 5 & 1 & 5 & 0 & 5 \\
\hline R18 & 4 & 4 & 4 & 0 & 4 \\
\hline R19 & 2 & 7 & 1 & 0 & 1 \\
\hline R20 & 8 & 9 & 5 & 0 & 5 \\
\hline
\end{tabular}

Tabela 4 : Resultado classe 2 - Módulo Gramatical

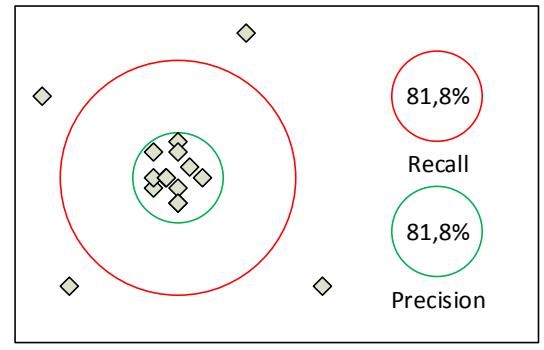

Figura 14 : Precision e Recall para Classe 2 - Módulo Gramatical

\subsection{Avaliação do resultado com Módulo Or- tográfico e Gramatical para classe 2}

Nesta etapa, todas as redações que passaram pela avaliação somente com o analisador gramatical foram novamente avaliadas, porém com o módulo ortográfico acoplado ao módulo gramatical. Com a união dos módulos, foi observado o comportamento e a dinâmica de ambos em encontrar e oferecer sugestões para os erros encontrados Os resultados obtidos são mostrados na Tabela 5 e Figura 15.

\begin{tabular}{l|lllll}
\hline Redações & $\begin{array}{c}\text { Erros } \\
\text { Gram. }\end{array}$ & $\begin{array}{c}\text { Erros } \\
\text { Ort. }\end{array}$ & $\begin{array}{c}\text { Correções } \\
\text { gramaticais }\end{array}$ & $\begin{array}{l}\text { Correções } \\
\text { sintáticas }\end{array}$ & Total \\
\hline R16 & 3 & 2 & 3 & 2 & 5 \\
R17 & 5 & 1 & 5 & 1 & 6 \\
R18 & 4 & 4 & 4 & 5 & 9 \\
R19 & 2 & 7 & 2 & 7 & 9 \\
R20 & 8 & 9 & 8 & 9 & 17 \\
\hline
\end{tabular}

Tabela 5 : Resultado classe 2 com módulos Ortográfico e Gramatical

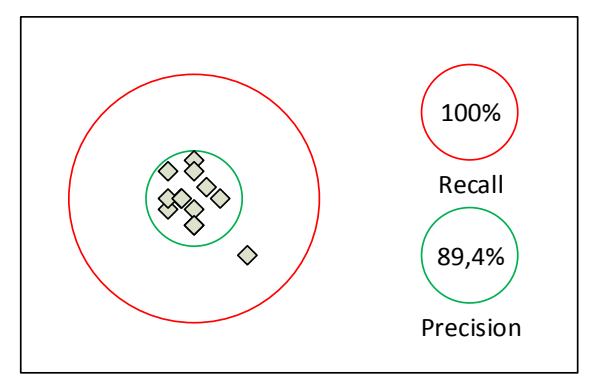

Figura 15 : Precision e Recall para Classe 2 - Módulos Ortográfico e Gramatical

\subsection{Discussão geral para os Módulos ortográ- fico e gramatical}

Com bases nos resultados, a maioria dos erros esperados foi encontrada.

Pode ser observado que $80 \%$ das redações foram corrigidas com sucesso. Quando o módulo ortográfico atuou em conjunto com o módulo gramatical, os erros encontrados foram os erros esperados. Valores de Precision e Recall muito altos podem ser explicados por ter uma base ainda muito pequena no dicionário auxiliar. Além do mais, é possível observar que a avaliação ortográfica é de extrema importância para uma boa avaliação gramatical.

O Classificador gramatical pode ter sido um fator que influenciou nos resultados de Recall, uma vez que, por utilizar dicionários, a base precisa ser absolutamente grande. Contudo, utilizando o Analisador sintático responsável pela análise gramatical das palavras, a base não necessitou ter todos os vocábulos da Língua Portuguesa.

É necessário o enriquecimento de técnicas de semântica lógica ainda para Analisadores Sintáticos para a Língua Portuguesa. Em relação aos demais idiomas, Parsers para Derivação de Árvores Sintáticas ainda demandam de um longo caminho para possuírem uma eficácia muito boa.

\section{Trabalhos Relacionados}

Nesta seção, serão apresentados os trabalhos que envolvem correções de atividades escritas, apresentando características referentes a cada um.

O CoGrOO Corretor Gramatical de código aberto capaz de identificar diversos erros relacionados à colocação pronominal, concordância nominal, concordância sujeito-verbo, uso da crase, dentre outros. Todos eles voltados para o idioma Português do Brasil [35]. O CoGrOO utiliza uma análise híbrida com o texto sendo mapeado logo de início com o uso de técnicas estatísticas de PLN - Processamento de Linguagem Natural, e em seguida, o sistema responsável por identificar os possíveis erros gramaticais é executado com base nas regras definidas.

LanguageTool É um sistema verificador de estilo e gramatical de código aberto. Foi desenvolvido primeiramente para a língua Inglesa, porém, atualmente realiza a correção gramatical para diferentes idiomas [37]. LanguageTool recebe como entrada o texto e retorna uma lista com os possíveis erros. Para a detecção dos erros, cada sentença é dividida em palavras, e essas palavras 
recebem sua tag part-of-speech ${ }^{14}$ [38] que estabelece a relação do discurso que esta palavra tem na frase. Cada frase é dividida em sintagmas nominais, unidades geradas por uma ou mais palavras que desempenham uma função sintática na frase [39]. Quando o erro é identificado, cada regra que deu origem ao erro possui uma explicação do erro cometido e a posição no qual o erro foi encontrado. As regras para expressar os erros descrevem o erro não somente ao nível de palavra, mas também ao nível de frase. Com a integração aos processadores de textos, o LanguageTool pode usar a verificação durante a entrada de texto, ou seja, a verificação on-the$\mathrm{fly}^{15}$. As regras são escritas e expressas em um arquivo XML, e pode ser facilmente alteradas pelos usuários.

ReGra é um revisor Gramatical para o idioma Português do Brasil. Foi desenvolvido com parceria USP-Itautec/Philco [40]. Embora tenha suporte a corretor ortográfico, o ReGra não inclui rotinas para este tipo de verificação.

Três módulos constituem o ReGra: Módulo Estatistico: Oferece tratamento estatístico realizado por uma série de cálculos, informando quantidade de parágrafos, sentenças, palavras. Esse módulo é o responsável por classificar o grau de dificuldade de leitura do texto. Módulo Mecânico: Verifica erros que não podem ser verificados por um corretor ortográfico, como palavras ou símbolos repetidos, balanceamento incorreto de delimitadores como parêntesis e aspas, entre outros. Módulo Gramatical: Neste módulo, é feito um levantamento de erros mais comuns entre usuários. Com esse levantamento, o objetivo foi implementar um módulo que voltasse para o interesse dos potenciais usuários.

Considerando correções léxico-sintáticas, nenhum dos trabalhos apresentados faz uso da correção léxica, e por se tratar de correções de atividades escritas, esse tipo de avaliação não pode ser esquecida. O Sistema proposto, além de cobrir erros gramaticais, também faz uso da correção ortográfica, cobrindo assim, o critério léxicosintático de correção.

\section{Conclusões}

Este trabalho mostrou e discutiu, através de resultados, técnicas de Processamento de Linguagem Natural para correção textual de Redações na língua Portuguesa de forma automática.

O módulo Ortográfico - Gramatical cumpriu com a primeira competência de avaliação proposta pelo INEP para avaliar os estudantes que fazem o ENEM.

\footnotetext{
${ }^{14}$ Etiquetador morfológico

${ }^{15}$ Correção dinâmica em tempo de escrita
}

Foi possível levantar um estudo, avaliando que palavras com ortografia errônea, podem atrapalhar o contexto de toda a frase, fazendo com que a correção gramatical gerada pelo analisador ignorasse erros gramaticais.

O Sistema proposto traz consigo um modelo de correção com as respectivas sugestões, ajudando ao aluno e acredita-se que atue diminuindo a sobrecarga por parte do professor, dado que as correções realizadas pelo sistema se assemelha as correções realizadas por um especialista. Com esse sistema de correção, os alunos saberão o que está errado e quais sugestões ele poderá usar para corrigir a palavra ou sentença, o que exige que o aluno efetue a correção manualmente, ponderando com base nas sugestões e localizações dos respectivos erros.

Com o principal objetivo de marcar as palavras como corretas ou não, o dicionário auxiliar necessita ainda de uma base maior de dados, para melhor classificação, pois, quando se encontra uma palavra correta, que não está presente na base de dados, o mesmo marca a palavra como um erro, o que justifica algumas correções realizadas a mais do que o que deveria ser feito. No módulo ortográfico, a base de dados do dicionário utilizado ainda é pequena, o que acarreta em uma correção errônea quando se tem uma nova palavra, e adição de palavras novas exige trabalho manual. No módulo gramatical, algumas regras lógicas que fazem a avaliação da sintática, como conjugações verbais ainda se encontram com pequenos erros de avaliação com a posição dos termos na oração, ou melhor, quanto à posição do sujeito e predicado.

O experimento realizado avaliou uma quantidade limitada de redações, visando o lado mais técnico do sistema. Um experimento com maior quantidade de redações e comparações com redações corrigidas com o controle de tempo por especialistas poderia ser aplicado.

A expansão do dicionário e uma implementação de um modelo de dicionário Semântico baseado em ontologias, facilitando estudos relacionados à semântica e à pragmática do Módulo Gramatical, podendo realizar não somente frases e sentenças, mas extrair significados utilizando a técnicas de Latent Semantic Analisys (LSA), visando uma correção da redação não somente avaliando termos ou expressões, mas todo o contexto. A Implementação de um componente que realize a adição de palavras ao dicionário auxiliar com base na existência de dados utilizando aprendizagem de máquina pode ser adicionada.

A continuação da pesquisa seguindo as competências do ENEM também será reavaliada. Evolução de módulos que fazem análises de contexto e correlação das sentenças com o tema proposto. 


\section{Referências}

[1] De Almeida, Maria Elizabeth Bianconcini. "Educação a distância e tecnologia: contribuições dos ambientes virtuais de aprendizado." Anais do Workshop de Informática na Escola. Vol. 1. No. 1. 2003.

[2] Da Silva, Antonio Carlos Ribeiro. "Educação a distância e o seu grande desafio: o aluno como sujeito de sua própria aprendizagem." 2004.

[3] Moran, José Manuel. A educação a distância e os modelos educacionais na formação dos professores. BONIN, Iara et al. Trajetórias e processos de ensinar e aprender: políticas e tecnologias. Porto Alegre: Edipucrs, p. 245-259, 2008.

[4] Sclater, Niall. Web 2.0, personal learning environments, and the future of learning management systems. Research Bulletin, v. 13, n. 13, p. 1-13, 2008.

[5] Sleeman, Derek; BROWN, John Seely. Intelligent tutoring systems. 1982.

[6] Brusilovsky, Peter. Methods and techniques of adaptive hypermedia. In: Adaptive hypertext and hypermedia. Springer Netherlands, p. 1-43. 1998.

[7] Stahl, Gerry; Koschmann, Timothy; SUTHERS, Dan. Computer-supported collaborative learning: An historical perspective. Cambridge handbook of the learning sciences, v. 2006, p. 409-426, 2006.

[8] Martin, Fred G. Will massive open online courses change how we teach?. Communications of the ACM, v.55, n.8, p.26-28, 2012.

[9] Harasim, Linda. Online education. Computer networking and scholarly communication in the twenty-first-century university, p. 203-214, 1996.

[10] Foley, B. \& Kobaissi, A. Using virtual chat to study in informal learning in online environments Annual Meeting of the American Educational Researcher Association, AERA, 2006.

[11] Vrasidas, Charalambos; Mcisaac, Marina Stock. Factors influencing interaction in an online course. American Journal of Distance Education, v. 13, n. 3, p. 22-36, 1999.

[12] Da Silva, Antonio Carlos Ribeiro. Educação a distância e o seu grande desafio: o aluno como sujeito de sua própria aprendizagem. 2004.

[13] Moran, José Manuel. Educação inovadora na Sociedade da Informação. ANPEDE. São Paulo, v. 168, n. 200.17, 2006.

[14] Santos, Jário; Paiva, Ranilson; Bittencourt, Ig. Avaliação automática de atividades escritas baseada em algoritmo genético e processamento de linguagem natural: Avaliador OrtográficoGramatical. Concurso de Teses e Dissertações em Informática na Educação. CBIE, p.95, 2015.

[15] Ilari, Rodolfo. Introdução ao estudo do léxico: brincando com as palavras. Editora Contexto, 2002.

[16] Oliveira, Fabio AD. Processamento de linguagem natural: princípios básicos e a implementação de um analisador sintático de sentenças da língua portuguesa. Porto Alegre, 2009.

[17] Martin, James H.; Jurafsky, Daniel. Speech and language processing. International Edition, 2000.

[18] Voutilainen, Atro. A syntax-based part-ofspeech analyser. In: Proceedings of the seventh conference on European chapter of the Association for Computational Linguistics. Morgan Kaufmann Publishers Inc., p. 157-164.1995.

[19] Chanod, Jean-Pierre; Tapanainen, Pasi. Creating a tagset, lexicon and guesser for a French tagger. arXiv preprint cmp-lg/9503004, 1995.

[20] Daelemans, Walter et al. MBT: A memorybased part of speech tagger-generator. arXiv preprint cmp-lg/9607012, 1996.

[21] Schmid, Helmut. Probabilistic part-of-speech tagging using decision trees. In: Proceedings of the international conference on new methods in language processing. p. 44-49. 1994.

[22] Ma, Q., K. Uchimoto, M. Murata, \& H. Isahara. Elastic neural networks for part of speech tagging. In International Joint Conference on Neural Networks(IJCNN'99), Washington, DC. 1999.

[23] Ratnaparkhi, A. A.. A maximum entropy partof-speech tagger. In Proceedings of the Empirical Methods in Natural Language Processing Conference, Philadelphia, Pa. 1996.

[24] Wilkens, M. \& J. Kupiec. Training hidden markov models for part of speech tagging. Revision 


\section{1996.}

[25] Brill, E. Some advances in transformation-based part of speech tagging. In AAAI '94: Proceedings of the twelfth national conference on Artificial intelligence (vol. 1), Seattle, Washington, United States, pp. 722-727. American Association for Artificial Intelligence. 1994.

[26] Brill, E. Transformation-based error-driven learning and natural language processing: A case study in part of speech tagging. Computational Linguistics 21(4), 543-565. 1995.

[27] Brill, E. Unsupervised learning of disambiguation rules for part of speech tagging. In Natural Language Processing Using Very Large Corpora. Kluwer Academic Press. 1997.

[28] Younger, Daniel H. Recognition and parsing of context-free languages in time n 3. Information and control, v. 10, n. 2, p. 189-208, 1967.

[29] Aho, Alfred V.; ULLMAN, Jeffrey D. The theory of parsing, translation, and compiling. Prentice-Hall, Inc., 1972.

[30] Deb, Kalyanmoy et al. A fast and elitist multiobjective genetic algorithm: NSGA-II. Evolutionary Computation, IEEE Transactions on, v. 6, n. 2, p. 182-197, 2002.

[31] Neumann, John von; BURKS, Arthur W. Theory of self-reproducing automata. 1966.

[32] Papadimitriou, Fivos. Modelling spatial landscape complexity using the Levenshtein algorithm. Ecological Informatics, v. 4, n. 1, p. 4855, 2009.

[33] Damerau, Fred J. A technique for computer detection and correction of spelling errors, Communications of the ACM (ACM) 7 (3): 171-176, 1964.

[34] Moschitti, Alessandro. Efficient convolution kernels for dependency and constituent syntactic trees. In: Machine Learning: ECML 2006. Springer Berlin Heidelberg, p. 318-329. 2006.

[35] Colen, William; Finger, Marcelo. Improving CoGrOO: the Brazilian Portuguese Grammar Checker. Em: STIL 2013 The 9th Brazilian Symposium in Information and Human Language Technology, 2013.

[36] Vladimir I. Levenshtein, Binary codes capable of correcting deletions, insertions, and reversals, Doklady Akademii Nauk SSSR, 163(4):845848, 1965 (Russian). English translation in
Soviet Physics Doklady, 10(8):707-710, 1966.

[37] Naber, Daniel. A rule-based styyle and grammar checker. PhD thesis, Faculty of Technology, Bielefeld University, 2003.

[38] Voutilainen, Atro. A syntax-based part-ofspeech analyser. In: Proceedings of the seventh conference on European chapter of the Association for Computational Linguistics. Morgan Kaufmann Publishers Inc., p. 157-164. 1995.

[39] Church, Kenneth Ward. A stochastic parts program and noun phrase parser for unrestricted text. In: Proceedings of the second conference on Applied natural language processing. Association for Computational Linguistics, p. 136143. 1988 .

[40] Nunes, M. e Oliveira, Osvaldo. O Processo de desenvolvimento do revisor gramatical ReGra. NILC - Núcleo Interinstitucional de Linguística Computacional, ICMC - USP, 2000. 